\title{
Relationships Between Bioactive Compound Content and the Antiplatelet and Antioxidant Activities of Six Allium Vegetable Species
}

\author{
Hebe Vanesa Beretta ${ }^{1}$, Florencia Bannoud ${ }^{1}$, Marina Insani ${ }^{2}$, Federico Berli $i^{1,3}$, \\ Pablo Hirschegger ${ }^{3}$, Claudio Rómulo Galmarini ${ }^{1,3,4}$ and Pablo Federico Cavagnaro ${ }^{1,3,4 *}$ \\ ${ }^{1}$ National Council of Scientific and Technical Research (CONICET), Av. Rivadavia 1917, \\ C1033AAJ Buenos Aires, Argentina \\ ${ }^{2}$ National Institute of Agricultural Technology (INTA), Nicolas Repetto y de los Reseros s/n, \\ Hurlingham, 1686 Buenos Aires, Argentina \\ ${ }^{3}$ National University of Cuyo, Faculty of Agricultural Sciences, Almirante Brown 500, Luján de Cuyo, \\ 5505 Mendoza, Argentina \\ ${ }^{4}$ National Institute of Agricultural Technology (INTA) - E.E.A. La Consulta, La Consulta CC8, \\ San Carlos, 5567 Mendoza, Argentina \\ Received: March 30, 2016 \\ Accepted: January 17, 2017
}

\begin{abstract}
Summary
Allium sp. vegetables are widely consumed for their characteristic flavour. Additionally, their consumption may provide protection against cardiovascular disease due to their antiplatelet and antioxidant activities. Although antiplatelet and antioxidant activities in Allium sp. are generally recognised, comparative studies of antiplatelet and antioxidant potency among the main Allium vegetable species are lacking. Also, the relationship between organosulfur and phenolic compounds and these biological activities has not been well established. In this study, the in vitro antiplatelet and antioxidant activities of the most widely consumed Allium species are characterised and compared. The species total organosulfur and phenolic content, and the HPLC profiles of 11 phenolic compounds were characterised and used to investigate the relationship between these compounds and antiplatelet and antioxidant activities. Furthermore, antiplatelet activities in chives and shallot have been characterised for the first time. Our results revealed that the strongest antiplatelet agents were garlic and shallot, whereas chives had the highest antioxidant activity. Leek and bunching onion had the weakest both biological activities. Significantly positive correlations were found between the in vitro antiplatelet activity and total organosulfur $(\mathrm{R}=0.74)$ and phenolic (TP) content $(R=0.73)$, as well as between the antioxidant activity and TP $(R=0.91)$ and total organosulfur content $(R=0.67)$. Six individual phenolic compounds were associated with the antioxidant activity, with catechin, epigallocatechin and epicatechin gallate having the strongest correlation values $(\mathrm{R}>0.80)$. Overall, our results suggest that both organosulfur and phenolic compounds contribute similarly to Allium antiplatelet activity, whereas phenolics, as a whole, are largely responsible for antioxidant activity, with broad variation observed among the contributions of individual phenolic compounds.
\end{abstract}

Key words: Allium sp., garlic, onion, antiplatelet activity, antioxidant activity, phenolic compounds

\footnotetext{
*Corresponding author: Phone: +54 2614605 737; E-mail: pablocavagnaro@hotmail.com

ORCiD IDs: 0000-0001-9245-5640 (Beretta), 0000-0002-4813-9429 (Bannoud), 0000-0003-1654-0549 (Insani), 0000-0003-1235-5901 (Berli), 0000-0001-5875-9640 (Hirschegger), 0000-0002-7958-0110 (Galmarini), 0000-0001-5838-0876 (Cavagnaro)
} 


\section{Introduction}

Cardiovascular disease (CVD) is the leading cause of death worldwide, with higher prevalence in developed countries. Foods with antiplatelet and antioxidant compounds can reduce the risk of CVD by maintaining haemostasis and aiding in the prevention of thrombosis and oxidative stress (1). Among Allium sp. functional properties that may confer protection against CVD, antiplatelet activity has been extensively studied in garlic (2-5) and onion (5-8), and in selected organosulfur compounds from these species (9). Antiplatelet agents have also been found in leek (10) and bunching onion (11). These studies used very different experimental conditions (e.g. different sources of platelets, agonists and aggregometer apparatus; and different sources of the antiplatelet agents, such as fresh or cooked plant materials, or isolated compounds, etc.) and they only analysed antiplatelet activity of a single Allium species, making their results not directly comparable. Thus, comparative analysis of the antiplatelet efficacy of the main Allium vegetables, using standardised experimental procedures, would be of great interest. Also, antiplatelet activity has not been investigated in chives and shallot. These Allium vegetables are economically important and widely consumed in specific regions of the world, such as chives in Asia, and shallot in parts of Europe and America.

Antioxidant activity has been reported in several $\mathrm{Al}$ lium vegetables, including onion $(12,13)$, garlic $(5,14)$, shallot $(15)$, leek $(13)$, chives $(15,16)$ and bunching onions (16). However, comparisons of antioxidant strength among these species, using the same experimental conditions, have not been reported.

Previous studies have suggested that organosulfur and phenolic compounds are, in part, responsible for $\mathrm{Al}$ lium antiplatelet activity. Briggs et al. (9) evaluated in vitro antiplatelet activity of four thiosulfinates (TSs) and found that all of them inhibited platelet aggregation, but their antiplatelet strength differed. In addition, combinations of different TSs revealed non-additive effects on in vitro antiplatelet activity, suggesting that the antiplatelet potential of Allium extracts cannot be predicted only by the presence and the treated dose of organosulfur components. Among the phenolic compounds, quercetin, the main polyphenol found in onions and shallots (17), inhibited platelet aggregation in vivo (18) and in vitro (19). The effect of other Allium phenolic compounds on platelet aggregation has not been reported to date.

Phenolic compounds have also been associated with Allium antioxidant activity. Total phenolic content was significantly and positively correlated with antioxidant activity in onion and shallot (13), garlic (14), and leek (20). Among Allium polyphenols, the flavonoid quercetin was further investigated, revealing strong antioxidant activity (21). Interestingly, quercetin is absent in garlic (22), which has been found to possess stronger antioxidant activity than onion (23), suggesting that compounds other than quercetin must be involved in garlic, and perhaps other alliums, antioxidant activity. Relationships between different phenolic compounds present in the main Allium vegetables and their antioxidant activity may shed light on the compound relative contribution to Allium antioxi- dant activity. High-performance liquid chromatography (HPLC) analysis is an ideal technique for this purpose, since it allows precise quantitative determination of several individual phenolic compounds simultaneously. In contrast, most of the previous Allium studies have used spectrophotometry-based determinations of total phenolics to establish associations between total phenolic content and antioxidant activity.

The goals of the present study are: (i) to perform comparative analyses of antiplatelet and antioxidant activities among the main Allium vegetable species, (ii) to investigate the antiplatelet activity of the so far uncharacterised Allium vegetables, (iii) to characterise the content of bioactive organosulfur and phenolic compounds in these species, and examine possible associations between these compounds and the functional properties.

The comparative compositional and functional characterisation of Allium species will provide objective information for promoting the consumption of some Allium vegetables on the basis of their nutraceutical value. Also, the association between individual compounds and the antiplatelet or antioxidant activities may provide rationale for further research aiming at establishing causative effects for selected compounds, for example, by testing antiplatelet or antioxidant activities in selected phenolics and estimating their relative contribution to the overall activity. Such information would be useful for breeding purposes, e.g. if compounds with large contribution to the trait were revealed, since rapid determinations of such compounds could be used as markers for the indirect selection of plants with high functional value.

\section{Materials and Methods}

\section{Plant materials}

Six Allium (Alliaceae) species were obtained from a vegetable market in Mendoza, Argentina. All the vegetables were produced locally and they appeared fresh, with no sign of oxidation or dehydration. For each species, three replicates were used. Each replicate consisted of 10 onion bulbs (mean $m$ (bulb)=85 g), 10 shallot bulbs (mean $m$ (shallot bulb)=40 g), 10 garlic cloves (one clove per bulb from 10 different bulbs; mean $m$ (clove)=2.5 g), 10 units of leek (white shaft) and 10 and $20 \mathrm{~g}$ of the edible tissues of chives (leaves) and bunching onion (leaves and white shaft), respectively.

\section{Processing of samples}

Sample processing and preparation of aqueous extracts were performed as previously described by Galmarini et al. (7). Briefly, the bulb and clove outer dry scales of onion, garlic and shallot were removed and they were cut in quarters longitudinally. The edible part of chives, bunching onions and leeks was cut in fine slices. Peeled bulbs and chopped fresh tissues of each Allium species were juiced in two volumes (by mass per volume) of distilled water, with the exception of chives, which was juiced in four volumes of distilled water, using a blender (model MR 400 Plus; Braun, Kronberg, Germany). 


\section{In vitro antiplatelet activity}

In vitro antiplatelet activity was determined in Allium extracts (without further dilution) as described previously for garlic (4) and onion (7) using an electrical impedance aggregometer (Chrono-Log, Havertown, PA, USA) in whole blood (24). Blood was collected from two healthy non-smoker donors, a male and a female, aged 30 and 25 , respectively, who had abstained from eating alliums or other known platelet-inhibitory foods for at least 5 days prior to venipuncture. For each Allium juice sample, three technical replicates were used (i.e. three determinations of in vitro antiplatelet activity per sample). In vitro antiplatelet activity was expressed as percentage of platelet aggregation inhibition compared to control samples prepared in the same way but without the addition of Allium juice. For each species, the juice concentration required to inhibit platelet aggregation by $50 \%\left(\mathrm{IC}_{50}\right)$ was estimated from dose-response curves constructed using different dosages of Allium juice vs. the percentage of aggregation inhibition. $\mathrm{IC}_{50}$ values were expressed in $\mathrm{mg}$ of fresh tissue per $\mathrm{mL}$ of whole blood.

\section{Antioxidant activity}

Antioxidant activity in Allium extracts was determined based on their oxygen radical absorbance capacity (ORAC) (25). For this purpose, the extracts were diluted $500 \times$ in $75 \mathrm{mM}$ potassium phosphate buffer $(\mathrm{pH}=7.0)$, and antioxidant activity was estimated using a microplate fluorometer (Fluoroskan Ascent ${ }^{\mathrm{TM}}$ FL; Thermo Fisher Scientific, Wilmington, DE, USA) according to Berli et al. (26). Antioxidant activity was expressed in $\mu \mathrm{mol}$ of Trolox equivalents (TE) per $100 \mathrm{~g}$ of fresh mass (fm). Two technical replicates were used for each Allium juice sample.

\section{Soluble and total solids}

Soluble solid (SS) content was determined with a refractometer (model ZGRC-200ATC; Científica Schonfel, Buenos Aires, Argentina) as previously described (7), and expressed in ${ }^{\circ}$ Brix. For determination of total solids, expressed in dry matter $(\mathrm{dm})$ content, Allium fresh tissues were weighed and dried in a stove at $60^{\circ} \mathrm{C}$ until constant mass was obtained, and expressed in percentage (\%) of $\mathrm{fm}$.

\section{Pyruvate analysis}

Pyruvate content was used as an estimator of Allium total organosulfur content (6). Pyruvate determinations were performed in a Beckman DU-530 UV-Vis spectrophotometer (Beckman Coulter Inc., Brea, CA, USA) as reported by Schwimmer and Weston (27) and expressed in mmol of pyruvate per $100 \mathrm{~g}$ of $\mathrm{fm}$.

\section{Total phenolics}

Total phenolic content was estimated as described previously by Singleton and Rossi (28) using a Beckman DU-530 UV-Vis spectrophotometer (Beckman Coulter), and expressed in $\mathrm{mg}$ of gallic acid equivalents (GAE) per $100 \mathrm{~g}$ of $\mathrm{fm}$.

\section{HPLC analysis of phenolic compounds}

The mass fraction of 11 phenolic compounds was estimated in all Allium species by high-performance liquid chromatography (HPLC) using an Agilent 1100 Series HPLC system (Agilent Technologies, Santa Clara, CA, USA) equipped with an autosampler (WPALS G1367A), binary pump (G1312A), degasser unit (G1397A), autosampler thermostat (ALS Therm G1330B) and column compartment (COLCOM G1361A). The analytical column was an ODS-Hypersil ${ }^{\mathrm{TM}}$ C18 column $(250 \mathrm{~mm} \times 4.6 \mathrm{~mm}$, particle size $5 \mu \mathrm{m}$; Thermo Fisher Scientific Inc, Waltham, MA, USA). Detection of flavan-3-ols was performed with an Agilent 1100 fluorescence detector (G1321A) at $\lambda=280$ (excitation) and $310 \mathrm{~nm}$ (emission) and of flavonols with an Agilent 1200 diode array and multiple wavelength detector (G1365B) at $\lambda=380 \mathrm{~nm}$. The mobile phase used for separation was a linear gradient of $0.1 \%$ trifluoroacetic acid and acetonitrile $/ 0.1 \%$ trifluoroacetic acid in a ratio of 80:20 (by volume). Calibration curves constructed from pure compounds of catechin aglicon, epigallocatechin gallate $(\mathrm{EGCg})$, epicatechin gallate $(\mathrm{ECg})$, epigallocatechin (EGC), rutin, myricetin aglicon, quercetin aglicon, kaempferol aglicon, chlorogenic acid, coumaric acid, ferulic acid and caffeic acid were used to estimate compound mass fraction. Quercetin, myricetin, catechin, kaempferol and ferulic acid glycosides were evaluated after chemical hydrolysis. The mass fraction of individual phenolic compounds was expressed in $\mathrm{mg}$ per $100 \mathrm{~g}$ of $\mathrm{fm}$. $\mathrm{ECg}$ and EGC are reported together as ECg+EGC.

\section{Total flavonoids and total non-flavonoid phenolics}

Total flavonoid (TF) content was calculated from HPLC data as the sum of the individual flavonoid compounds present in each Allium species (i.e. $\mathrm{TF}=$ quercetin+ catechin+EGCg+ECg+EGC+rutin+myricetin+kaempferol) and expressed in $\mathrm{mg}$ per $100 \mathrm{~g}$ of $\mathrm{fm}$. Total non-flavonoid phenolic (TNFP) content was calculated from HPLC data as the sum of the individual non-flavonoid phenolics present in each Allium species (i.e. chlorogenic acid+coumaric acid+ferulic acid+caffeic acid) and expressed in $\mathrm{mg}$ per $100 \mathrm{~g} \mathrm{fm}$.

\section{Thiosulfinate analysis}

Thiosulfinate content was determined spectrophotometrically using a Beckman DU-530 UV-Vis spectrophotometer (Beckman Coulter) according to Han et al. (29), and expressed in mmol TS per $100 \mathrm{~g} \mathrm{fm}$.

\section{Statistical analyses}

Single-factor and multifactor analyses of variance (ANOVA), mean value comparisons, Pearson's correlation analysis and principal component analysis (PCA) were performed using InfoStat software v. 4.0 for Windows (30). Mean value comparisons were performed by the least significant difference (Fisher's LSD) test, and p-value $<0.05$ was considered significant.

\section{Results and Discussion}

\section{Variation in antiplatelet activity among Allium vegetables}

The in vitro responses of human platelets to dosages of six Allium vegetables were measured and compared. All Allium species exhibited significant antiaggregatory 
effects, as compared to their negative controls. Antiplatelet activity varied significantly and broadly (more than 30-fold) among the six analysed Allium species, as indicated by their $\mathrm{IC}_{50}$ values (Table 1). In both blood donors, garlic was the most potent platelet inhibitor, followed by shallot and chives, whereas leek and bunching onion were the weakest antiplatelet agents. Garlic antiplatelet activity was approx. 5-, 13-, 23-, 31- and 32-fold higher than of shallot, chives, onion, bunching onion and leek, respectively, in both blood samples (Table 1).

In vitro antiplatelet activity of all the species varied in a dose-dependent manner (data not presented). Extracts of garlic and shallot inhibited platelet aggregation at all tested doses $(\mathrm{p}<0.05)$, beginning at 5 and $15-50 \mu \mathrm{L}$ per $\mathrm{mL}$ of blood, respectively. The minimum platelet inhibitory doses of other Allium extracts were 40- to 60-fold higher (i.e. 200-300 $\mu \mathrm{L}$ per $\mathrm{mL}$ of blood) than of garlic.

Antiplatelet activity of chives and shallot has not been investigated previously. In the present study, we report for the first time a significant in vitro antiplatelet activity induced by aqueous extracts of these two Allium vegetables. Our comparative analysis revealed that shallot and chives are, after garlic, potent antiaggregant agents (Table $1)$.

Although experimental conditions varied greatly among previously reported studies, and therefore results cannot be directly compared, the $\mathrm{IC}_{50}$ values presented in this study of garlic and onion are in general agreement with those from a previous report of Ali et al. (31) using platelet-rich plasma (PRP) instead of whole blood for assessing in vitro aggregometry. In their study, antiplatelet activity of the two species varied approx. 16-fold, with mean $\mathrm{IC}_{50}$ values of garlic and onion of 6.6 and $90 \mathrm{mg} / \mathrm{mL}$ of PRP, respectively, whereas in the present study garlic antiplatelet activity was approx. 22-fold higher than onion antiplatelet activity, with mean $\mathrm{IC}_{50}$ values of 3.6 and $81.7 \mathrm{mg}$ fm per $\mathrm{mL}$ of whole blood, respectively (Table 1 ).

Antiplatelet activities reported herein of garlic and onion are also comparable with values found for both species in our previous studies using the same in vitro antiplatelet activity procedure $(4,7,8,32,33)$. In these studies, garlic in vitro antiplatelet activity $(4,32)$ was much stronger than that of onion $(7,8,33)$, on fresh mass basis. Such differences in in vitro antiplatelet activity between the two species are likely due to their compositional variation, and their bulb water content. Garlic has higher content of total organosulfur compounds (Table 1) and different profile of these sulfur constituents, allicin being its predominant thiosulfinate (accounting for approx. 60-95\% of total thiosulfinates in fresh garlic) (5). Allicin, which is absent in onion, was found to be the most potent antiplatelet Allium thiosulfinate, as evaluated in vitro (9). Water content in the edible parts of the two most important Allium vegetables may also account for the observed differences in the amount of antiplatelet compounds (e.g. thiosulfinates) and therefore antiplatelet activity. While most onion cultivars have approx. 90-95\% water (by mass) in their bulbs $(7,33)$, garlic usually has water content in the range of 55-65\% (33). Thus, on fresh mass basis, the antiplatelet compounds in onion are much more diluted than in garlic, and this difference likely reflects part of the observed variation in in vitro antiplatelet activ- ity between the two species. Presumably, and more broadly, the observed variation in in vitro antiplatelet activity among the six analysed Allium vegetables may be due to the species differences in water content in their edible parts, the type and content of antiplatelet compounds (e.g. thiosulfinates and phenolic compounds) and their interactions.

Although in the present study no 'donor $\times$ in vitro antiplatelet activity' interaction was found, significant differences in platelet aggregation by onion and shallot were observed between blood donors (Table 1). In vitro antiplatelet activity induced by extracts of onion and shallot was weaker in the blood of donor 2 than of donor 1. Variation in the in vitro antiplatelet activity among blood donors induced by onion extracts has been reported previously (7).

\section{Variation of antioxidant activity among Allium species}

The present study constitutes the first comparative analysis of antioxidant activity among the main Allium vegetable species using the same experimental conditions (ORAC). Antioxidant activity was found in all Allium vegetables, with a significant variation among the species. Antioxidant activity mean values varied more than 8 -fold and ranged from 247.5 (in leek) to $2088.2 \mu \mathrm{mol}$ TE per 100 $\mathrm{g} \mathrm{fm}$ (in chives) (Table 1). The decreasing order for antioxidant strength was chives $>$ shallot $>$ garlic $>$ onion $>$ bunching onion>leek. Chives were significantly more potent antioxidants than all the other Allium species $(\mathrm{p}<0.05)$, with antioxidant activity values 5.8- to 8.4-fold higher than the weakest antioxidants; onion, bunching onion and leek (Table 1).

Our results are in accordance with a previous study by Morales-Soto et al. (34) reporting antioxidant ranges (as Trolox equivalents) on dry mass basis of 830-4300 and 520-13 $000 \mu \mathrm{mol} / 100 \mathrm{~g}$ for garlic and onion, respectively. For leek, ORAC ranged from 27 to $88 \mu \mathrm{mol}$ per $100 \mathrm{~g}$, whereas in bunching onions values of approx. $50 \mu \mathrm{mol}$ per $g$ were reported (35). The ranges of antioxidant activity previously reported for leek and bunching onion are slightly higher than the values found in these species in the present study. These discrepancies could be due to the compositional differences among the assayed cultivars, water content and agricultural practices used for growing the plant materials.

\section{Relationships between Allium functional properties and their bioactive compound content}

Data from compositional analysis of edible parts of six Allium vegetables are presented in Table 1. In order to explore relationships between the content of bioactive compounds and Allium antioxidant and antiplatelet activities, correlation analyses were performed among in vitro antiplatelet activity, antioxidant activity and phytochemical mass fraction (Tables 2 and 3). Significantly positive correlations were found between in vitro antiplatelet activity and pyruvate $(\mathrm{R}=0.74)$, total phenolics $(\mathrm{R}=0.73)$ and thiosulfinates $(\mathrm{R}=0.60)$, whereas no association was found between the antiplatelet activity and total non-flavonoid phenolics (TNFP) (Table 2).

The differences found in the antiaggregatory activity are likely due to compositional variations in antiplatelet 


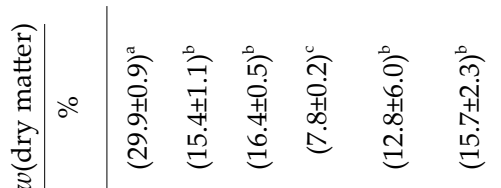

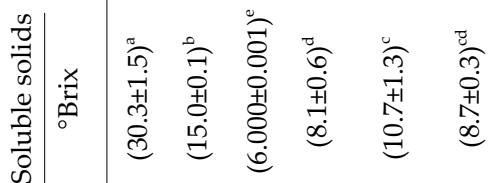

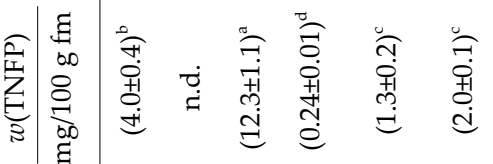

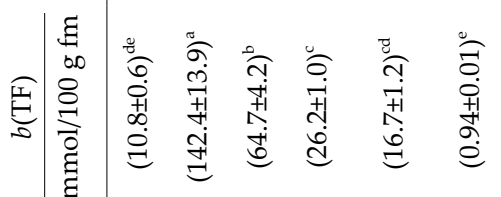

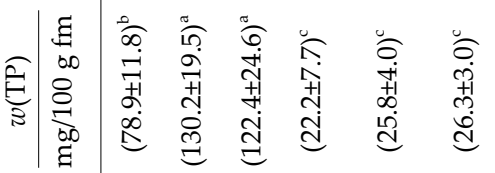

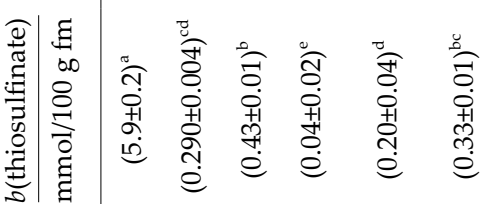

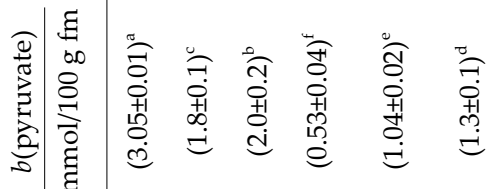

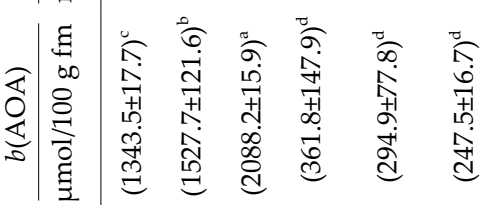

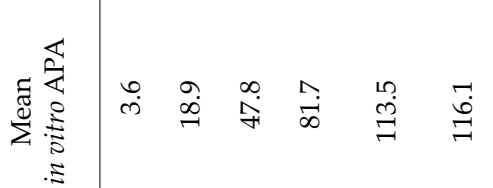

«ำ

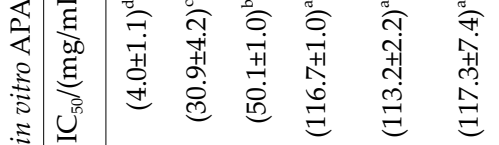

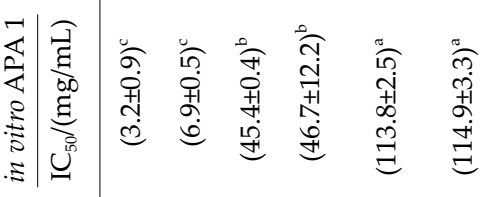

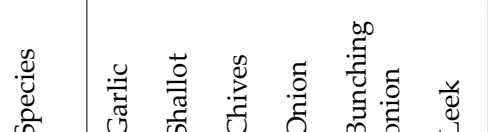

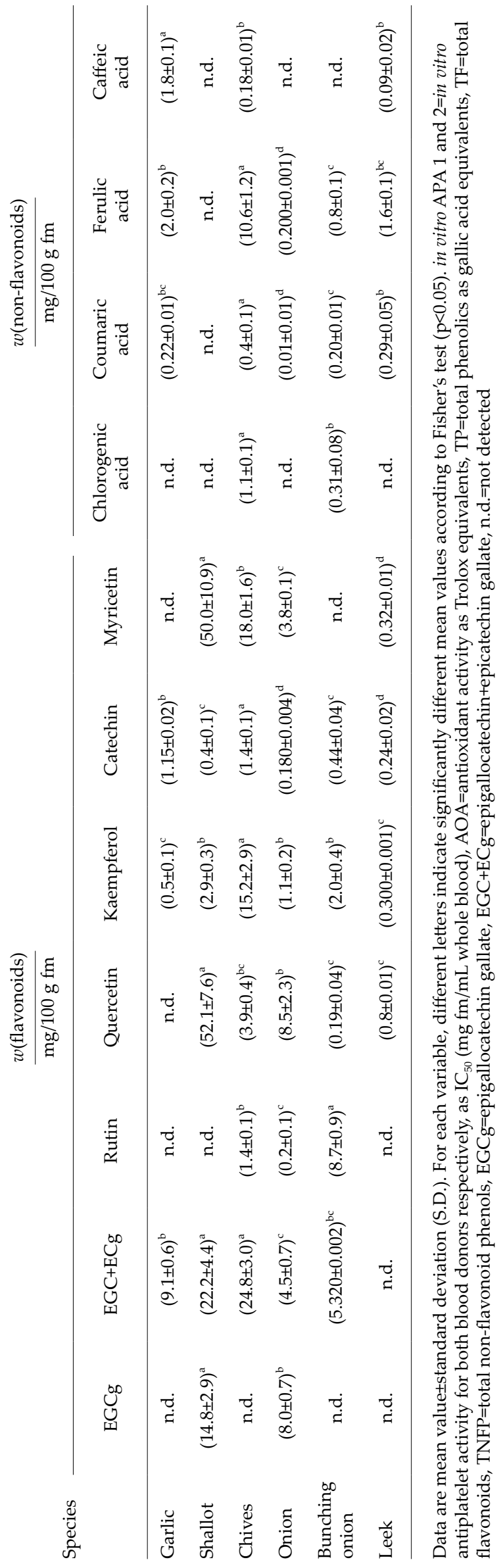



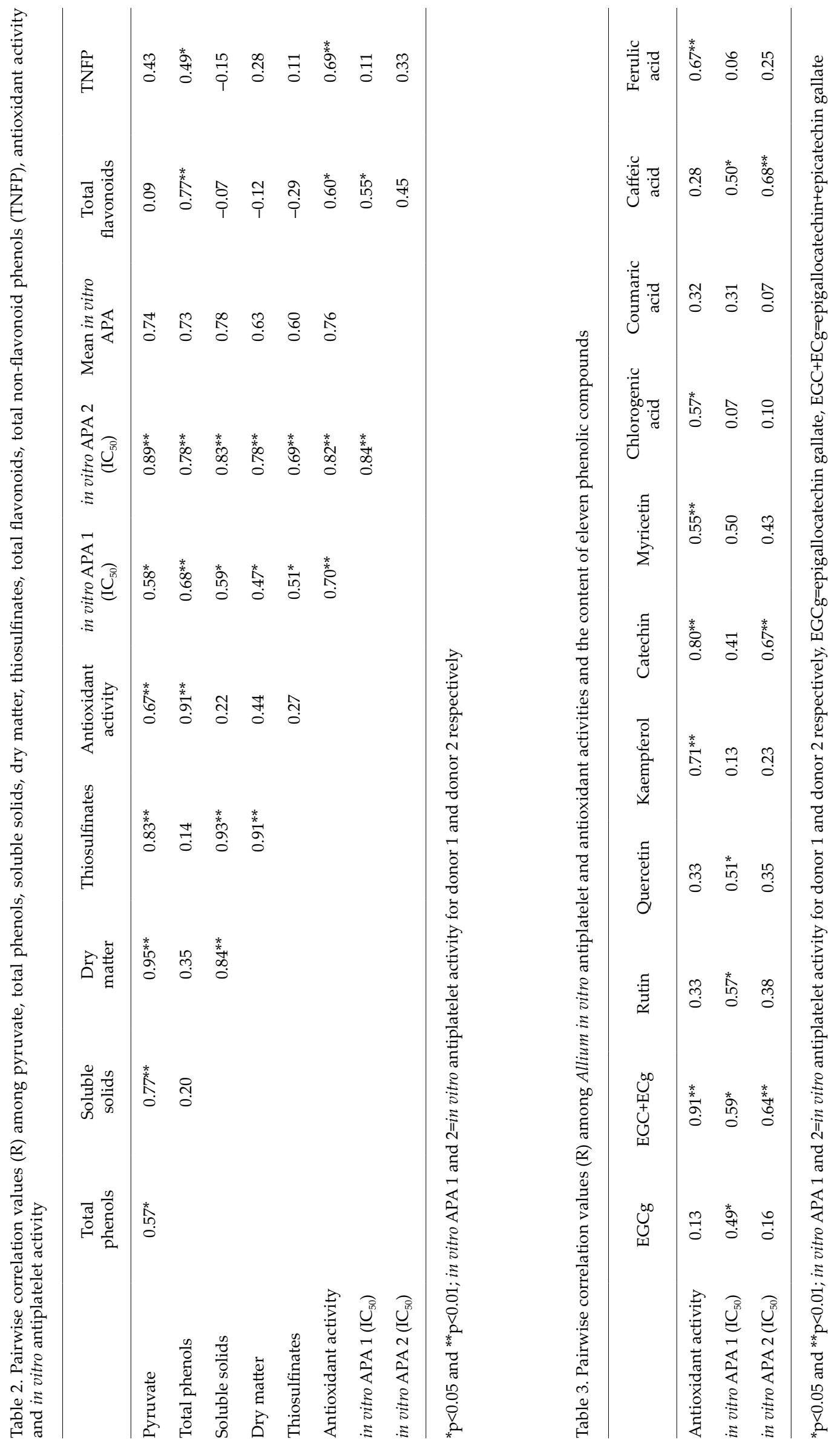
compounds among the examined Allium samples. Allium species differ in their organosulfur profiles, both in content and compound type, and isolated thiosulfinates from different alliums have proved different in vitro antiplatelet activity (9). In the present study, garlic was the most potent anti-aggregant agent, with in vitro antiplatelet activity values 6- to 40 -fold higher than any other Allium species. Also, garlic had significantly higher pyruvate (an estimator of total organosulfur compounds) and thiosulfinate content than the other species. These data, together with the positive correlations found between in vitro antiplatelet activity and pyruvate $(\mathrm{R}=0.74)$, and in vitro antiplatelet activity and thiosulfinates $(R=0.60)$ suggest that organosulfur compounds are involved in Allium in vitro antiplatelet activity.

Antiplatelet activity of phenolic compounds, such as some flavonoids, has also been reported (18). In this study significant positive correlations between in vitro antiplatelet activity and total phenolics $(\mathrm{R}=0.78)$, and between in vitro antiplatelet activity and six individual polyphenols $(\mathrm{R}=0.49-0.68)$ were found, suggesting that phenolic compounds are also involved in Allium antiplatelet activity. The strongest associations were found with caffeic acid $(\mathrm{R}=0.68)$, catechin $(\mathrm{R}=0.67)$, and EGC+ECg $(\mathrm{R}=0.59-0.64)$ (Table 3). Altogether, our data suggest that both organosulfur and phenolic compounds are involved in Allium in vitro antiplatelet activity. In addition, it is possible that interactions among some of these compounds may play an important role in determining the overall antiplatelet activity of each species.

Allium antioxidant activity was strongly and positively correlated with total phenolics $(\mathrm{R}=0.91, \mathrm{p}<0.001)$, suggesting that phenolic compounds are extensively involved in Allium antioxidant properties. Similarly, antioxidant activity was also positively associated with total phenolics $(\mathrm{R}=0.60)$ and TNFP $(\mathrm{R}=0.69)$ (Tables 2 and 3$)$. These results are in accordance with previous studies in onion and shallot (13), garlic (14), and leek (20), reporting significantly positive correlations between total phenolic content and antioxidant activity.

Further association analyses among individual polyphenols and antioxidant activity were performed. Significant positive correlations were found between antioxidant activity and EGC+ECg $(\mathrm{R}=0.91)$, catechin $(\mathrm{R}=0.80)$, kaempferol ( $R=0.71)$, ferulic acid $(R=0.67)$, chlorogenic acid $(R=0.57)$ and myricetin $(\mathrm{R}=0.55)$. Chives, the most potent antioxidant species, had significantly higher values of EGC+ECg, catechin, kaempferol, ferulic acid and chlorogenic acid than all other Allium vegetables (Table 1).

Although the most potent antioxidant species were chives and shallot, their phenolic profiles varied greatly (Table 1), suggesting that individual phenolic compounds differ in antioxidant strength. Balasundram et al. (35) proposed that the antioxidant activity of phenolic compounds depends on their chemical structure, in particular the number and positions of the hydroxyl groups and the nature of substitutions on the aromatic rings. Our results suggest that antioxidant activity in Allium depends not only on the chemical structure, but also on the mass fraction of each phenolic compound and on the combinations and interactions among them. Future studies regarding the antioxidant effects of individual phenolic compounds and of their interactions would be very useful to clarify the individual contribution of each compound to the overall antioxidant activity in each species.

In addition to the apparent role of polyphenols in $\mathrm{Al}$ lium antioxidant activity, we found significant correlation between the antioxidant activity and pyruvate $(\mathrm{R}=0.67)$ (Table 2), suggesting that organosulfur compounds may also contribute to this nutraceutical property. However, no significant correlation was found between the antioxidant activity and thiosulfinate content. These results contradict those of Block (5) reporting that allicin and other thiosulfinate derivatives have antioxidant properties. Further studies aiming at elucidating the contribution of thiosulfinates in Allium antioxidant activity are needed.

\section{Principal component analysis}

Principal component analysis (PCA) was applied on the whole data set of six Allium species. The dimensionality of the data was reduced from 8 partially correlated variables to 2 uncorrelated principal components (PC), PC1 and PC2, accounting for $84.2 \%$ of the observed variation. The loadings, eigenvalues and percentage of cumulative variance are presented in Table 4. PC1 correlates

Table 4. Loadings, eigenvalues and percentage of cumulative variance for the first two principal components of the whole data set (above) for six Allium species and the relative proportions of phenolic compounds (below)

\begin{tabular}{lrr}
\hline Variable & PC1 & PC2 \\
\hline in vitro APA 2 & 0.41 & -0.13 \\
Pyruvate & -0.39 & -0.09 \\
Dry matter & -0.35 & -0.29 \\
Antioxidant activity & -0.34 & 0.47 \\
in vitro APA 1 & 0.33 & -0.21 \\
Total phenolics & -0.32 & 0.53 \\
Thiosulfinates & -0.31 & -0.43 \\
Soluble solids & -0.30 & -0.40 \\
Total flavonoids & -0.15 & 0.50 \\
Total non-flavonoid phenols & -0.17 & 0.20 \\
Cumulative variance/\% & 58.9 & 84.2 \\
\hline Variable & PC1 & PC2 \\
\hline Quercetin & 0.45 & -0.19 \\
Catechin & -0.43 & -0.24 \\
EGCg & 0.37 & -0.17 \\
Ferulic acid & -0.34 & -0.26 \\
Myricetin & 0.33 & -0.12 \\
Coumaric acid & -0.31 & -0.28 \\
Caffeic acid & -0.30 & -0.21 \\
Kaempferol & -0.16 & 0.34 \\
EGC+ECg & -0.14 & 0.17 \\
Rutin & -0.07 & 0.46 \\
Chlorogenic acid & -0.07 & 0.56 \\
Cumulative variance/\% & 39.3 & 66.2 \\
\hline
\end{tabular}

PC1=principal component $1, \mathrm{PC} 2=$ principal component 2 , in vitro APA 1 and $2=$ in vitro antiplatelet activity for donor 1 and donor 2, EGCg=epigallocatechin gallate, EGC+ECg=epigallocatechin+ epicatechin gallate 
positively with in vitro antiplatelet activity and negatively with the following variables, in decreasing order: pyruvate, dry matter, antioxidant capacity, total phenols, thiosulfinates, soluble solids, and total flavonoids. PC2 correlates with total phenolic compounds, total flavonoids and antioxidant activity. The variation of the data is explained mainly by in vitro antiplatelet activity, pyruvate, dry matter, thiosulfinates and soluble solid content, and to a lesser extent by total phenolics and antioxidant activity. The graphic representation of the scores and loadings is presented in Fig. 1a, revealing a clear separation of the analysed Allium vegetables. Garlic is located in the bottom left side of the plot, which is characterised by high contents of pyruvate, solids and thiosulfinates, and low values of $\mathrm{IC}_{50}$ (i.e. lower $\mathrm{IC}_{50}$ values indicate higher in vitro antiplatelet activity). Onion, leek and bunching onion are located in the right bottom side, presenting high $\mathrm{IC}_{50}$ and low pyruvate, soluble solids, dry matter and thiosulfinate values. Shallot and chives were clearly differentiated from the other species by their high antioxidant capacity and total phenolic content. The PCA illustrated the strong correlations observed between antioxidant activity and total phenolics, and between in vitro antiplatelet activity and pyruvate, soluble solids, dry matter and thiosulfinates (Table 2). Concordantly, the fact that garlic and chives, the most potent antiplatelet and antioxidant species respectively, are situated in opposite quadrants of the plot suggests that organosulfur and phenolic compounds contribute different to Allium antiplatelet and antioxidant activities.

Fig. $1 \mathrm{~b}$ presents PCA of phenolic profiles of the six Allium species. Eleven partially correlated variables were reduced to 2 uncorrelated principal components, PC1 and PC2, accounting for $66 \%$ of the variation. PC1 mainly correlates with quercetin, catechin, EGCg, ferulic acid and myricetin. PC2 correlates with chlorogenic acid, rutin and kaempferol. In general, the PCA revealed a clear separation of the species according to their phenolic profiles. A clear separation of the studied species was observed. Shallot and onion were located in the bottom right side of the plot, which is characterised by high quercetin and EGCg content, whereas garlic and leek were in the bottom left side, characterised by high levels of catechin. Chives and bunching onion were located in the upper side of the plot, characterised by high mass fraction of chlorogenic acid, rutin and kaempferol.

\section{Conclusions}

Under our experimental conditions garlic and shallot were the most potent antiplatelet agents, consistently in the blood of both donors, whereas chives and shallot had the strongest antioxidant activities. Leek, bunching onion and onion were, in that order, the weakest antiplatelet and antioxidant agents. The present study has character-
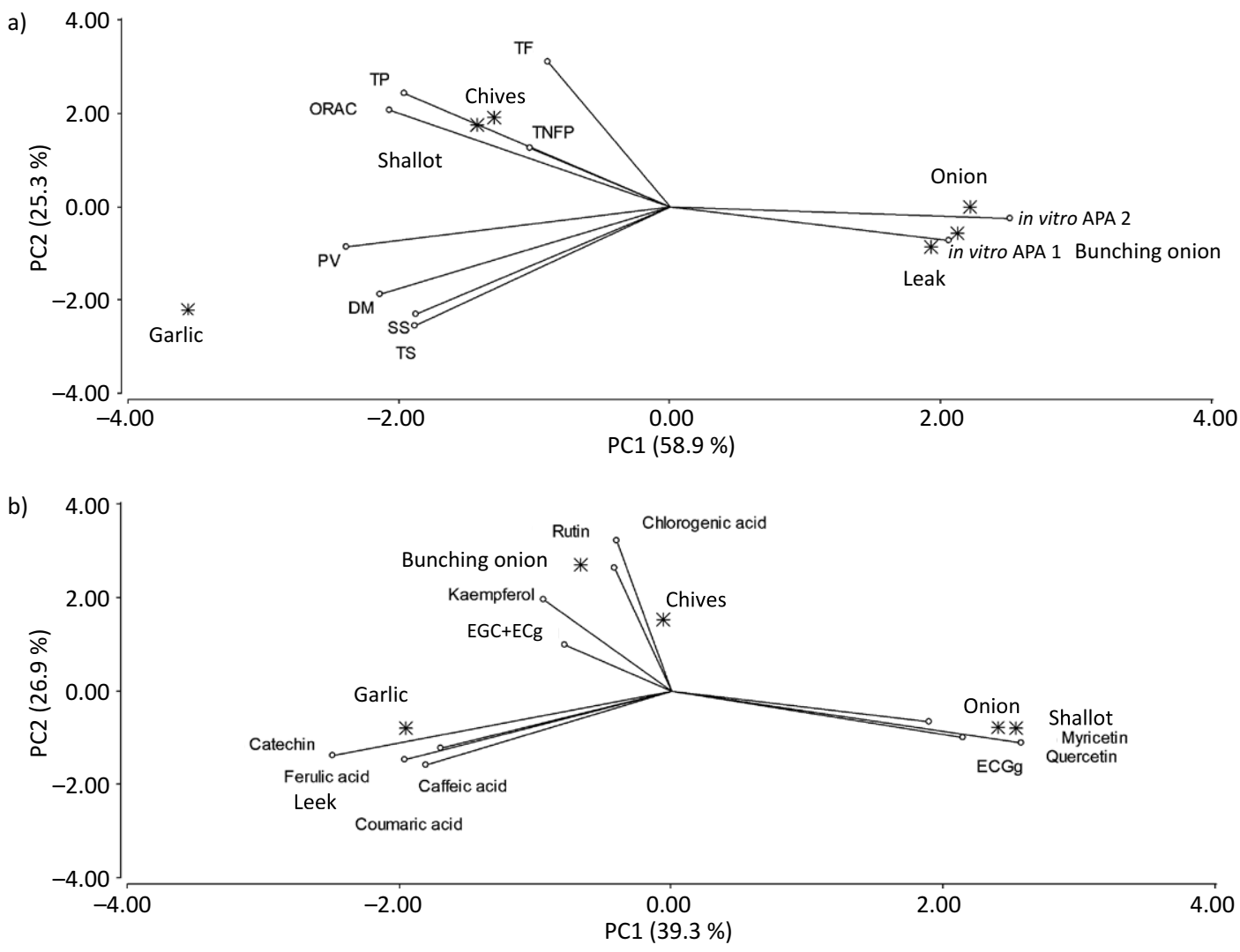

Fig. 1. Principal component analysis (PCA) of Allium functional properties and phytochemical content: a) PCA included the following variables: pyruvate (PV), total phenolics (TP), soluble solids (SS), dry matter (DM), thiosulfinates (TS), total flavonoids (TF), total non-flavonoid phenolics (TNFP), antioxidant activity measured by the oxygen radical absorbance capacity (ORAC), in vitro antiplatelet activity (APA) of both donors expressed as $\mathrm{IC}_{50} 1$ and 2, respectively; b) PCA of the phenolic profiles expressed as relative proportion (\%). EGCg=epigallocatechin gallate, EGC+ECg=epigallocatechin+epicatechin gallate 
ised for the first time in vitro antiplatelet activity of chives and shallot. Interestingly, shallot was one of the most potent species with both biological activities (antiplatelet and antioxidant activities).

Our results of correlation analyses strongly suggest that organosulfur and phenolic compounds are involved, to different extents, in both functional properties. While organosulfur and phenolic compounds contribute to similar extents in Allium in vitro antiplatelet activity, phenolics as a whole were largely responsible for antioxidant activity, with catechin, epigallocatechin and epicatechin gallate being the individual phenolics with strongest association with Allium antioxidant activity.

\section{Acknowledgements}

This study was funded by grant INTA-BID PICT-069. The authors gratefully acknowledge the blood donors that participated in the study.

\section{References}

1. McEwen BJ. The influence of diet and nutrients on platelet function. Semin Thromb Hemost. 2014;40:214-26. https://doi.org/10.1055/s-0034-1365839

2. Ariga T, Oshiba W, Tamada T. Platelet aggregation inhibitor in garlic. Lancet. 1981;317:150-1. https://doi.org/10.1016/S0140-6736(81)90729-7

3. Bordia A. Effect of garlic on human platelet aggregation in vitro. Atherosclerosis.1978;30:355-60. https://doi.org/10.1016/0021-9150(78)90129-6

4. Cavagnaro PF, Camargo A, Galmarini CR, Simon PW. Effect of cooking on garlic (Allium sativum L.) antiplatelet activity and thiosulfinates content. J Agric Food Chem. 2007;55:12808 . https://doi.org/10.1021/jf062587s

5. Block E. Garlic and other alliums: the lore and the science. Cambridge, UK: RSC Publishing; 2010.

6. Goldman IL, Schwartz BS, Kopelberg M. Variability in blood platelet inhibitory activity of Allium (Alliaceae) species accessions. Am J Bot. 1995;82:827-32. https://doi.org/10.2307/2445968

7. Galmarini CR, Goldman IL, Havey MJ. Genetic analyses of correlated solids, flavor, and health-enhancing traits in onion (Allium cepa L.). Mol Genet Genomics. 2001;265:543-51. https://doi.org/10.1007/s004380100445

8. Cavagnaro PF, Galmarini CR. Effect of processing and cooking conditions on onion (Allium cepa L.) induced antiplatelet activity and thiosulfinate content. J Agric Food Chem. 2012;60:8731-7.

https://doi.org/10.1021/jf301793b

9. Briggs W, Xiao H, Parkin KL, Shen C, Goldman IL. Differential inhibition of human platelet aggregation by selected Allium thiosulfinates. J Agric Food Chem. 2000;48:5731-5. https://doi.org/10.1021/jf0004412

10. Liakopoulou-Kyrakdes M, Sinakos Z. A low molecular weight peptide from Allium porum with inhibitory activity on platelet aggregation in vitro. Biochem Int. 1992;28:373-8.

11. Chen JH, Chen HI, Tsai SJ, Jen CJ. Chronic consumption of raw but not boiled Welsh onion juice inhibits rat platelet function. J Nutr. 2000;13:34-7.

12. Lee EJ, Patil BS, Yoo KS. Antioxidants of 15 onions with white, yellow, and red colors and their relationship with pungency, anthocyanin, and quercetin. LWT - Food Sci Tech- nol. 2015;63:108-14.

https://doi.org/10.1016/j.lwt.2015.03.028

13. Lu X, Wang J, Al-Qadiri HM, Ross CF, Powers JR, Tang J, Rasco BA. Determination of total phenolic content and antioxidant capacity of onion (Allium cepa L.) and shallot (Allium oschaninii) using infrared spectroscopy. Food Chem. 2011; 129:637-44. https://doi.org/10.1016/j.foodchem.2011.04.105

14. Queiroz YS, Ishimoto EY, Bastos DHM, Sampaio GR, Torres EY. Garlic (Allium sativum L.) and ready-to-eat garlic products: in vitro antioxidant activity. Food Chem. 2009;115:3714 . https://doi.org/10.1016/j.foodchem.2008.11.105

15. Štajner D, Popović BM, Calić-Dragosavac D, Malenčić D, Zdravković-Korać S. Comparative study on Allium schoenoprasum cultivated plant and Allium schoenoprasum tissue culture organs antioxidant status. Phytother Res. 2011;25: 1618-22. https://doi.org/10.1002/ptr.3394

16. Štajner D, Igić R, Popović BM, Malenčić D. Comparative study of antioxidant properties of wild growing and cultivated Allium species. Phytother Res. 2008;22:113-7. https://doi.org/10.1002/ptr.2278

17. Leighton T, Ginther C, Fluss L, Harter William K, Cansado J, Notario V. Molecular characterization of quercetin and quercetin glycosides in Allium vegetables. In: Huang MT, Ho CT, Lee CY, editors. Phenolic compounds in food and their effects on health II. Washington, DC, USA: ACS Publications; 1992; pp. 220-38.

https://doi.org/10.1021/bk-1992-0507.ch016

18. Hubbard GF, Wolffram S, de Vos R, Bovy A, Gibbins JM, Lovegrove JA. Ingestion of onion soup high in quercetin inhibits platelet aggregation and essential components of the collagen-stimulated platelet activation pathway in man: a pilot study. Br J Nutr. 2006;96:482-8.

https://doi.org/10.1079/bjn20061831

19. Hubbard GP, Stevens JM, Cicmil M, Sage T, Jordan PA, Williams CM, et al. Quercetin inhibits collagen-stimulated platelet activation through inhibition of multiple components of the glycoprotein VI signaling pathway. J Thromb Haemost. 2003;1:1079-88. https://doi.org/10.1046/j.1538-7836.2003.00212.x

20. Bernaert N, De Paepe D, Bouten C, De Clercq H, Stewart D, Van Bockstaele E, et al. Antioxidant capacity, total phenolic and ascorbate content as a function of the genetic diversity of leek (Allium ampeloprasum var. porrum). Food Chem. 2012; 134:669-77.

https://doi.org/10.1016/j.foodchem.2012.02.159

21. Zhang L, Ravipati AS, Koyyalamudi SR, Jeong SC, Reddy N, Smith PT, et al. Antioxidant and anti-inflammatory activities of selected medicinal plants containing phenolic and flavonoid compounds. J Agric Food Chem. 2011;59:12361-7. https://doi.org/10.1021/jf03146e

22. Sultana B, Anwar F. Flavonols (kaempeferol, quercetin, myricetin) contents of selected fruits, vegetables and medicinal plants. Food Chem. 2008;108:879-84. https://doi.org/10.1016/j.foodchem.2007.11.053

23. Benkeblia N. Free-radical scavenging capacity and antioxidant properties of some selected onions (Allium cepa L.) and garlic (Allium sativum L.) extracts. Braz Arch Biol Technol. 2005;48:753-9. https://doi.org/10.1590/S1516-89132005000600011

24. Cardinal DC, Flower RJ. The electronic aggregometer: a novel device for assessing platelet behavior in blood. J Pharmacol Methods. 1980;3:135-58. https://doi.org/10.1016/0160-5402(80)90024-8

25. Prior RL, Hoang H, Gu L, Wu X, Bacchiocca M, Howard L, et al. Assays for hydrophilic and lipophilic antioxidant capaci- 
ty (oxygen radical absorbance capacity $\left(\mathrm{ORAC}_{\mathrm{FL}}\right)$ ) of plasma and other biological and food samples. J Agric Food Chem. 2003;51:3273-9.

https://doi.org/10.1021/jf0262256

26. Berli FJ, Alonso R, Beltrano J, Bottini R. High-altitude solar UV-B and abscisic acid sprays increase grape berry antioxidant capacity. Am J Enol Vitic. 2015;66:65-72. https://doi.org/10.5344/ajev.2014.14067

27. Schwimmer S, Weston WJ. Onion flavor and odor, enzymatic development of pyruvic acid in onion as a measure of pungency. J Agric Food Chem. 1961;9:301-4. https://doi.org/10.1021/jf60116a018

28. Singleton VL, Rossi JA. Colorimetry of total phenolics with phosphomolybdic-phosphotungstic acid reagents. Am J Enol Vit. 1965;16:144-58.

29. Han J, Lawson L, Han G, Han P. A spectrophotometric method for quantitative determination of allicin and total garlic thiosulfinates. Anal Biochem. 1995:225:157-60. https://doi.org/10.1006/abio.1995.1124

30. Di Rienzo J, Casanoves F, Balzarini M, Gonzalez L, Tablada M, Robledo CW. InfoStat v. 2013. Cordoba, Argentina: Faculty of Agriculture, National University of Cordoba; 2013. Available from: http://www.infostat.com.ar.
31. Ali M, Bordia T, Mustafa T. Effect of raw versus boiled aqueous extract of garlic and onion on platelet aggregation. Prostaglandins Leukot Essent Fatty Acids. 1999;60:43-7. https://doi.org/10.1054/plef.1998.0006

32. Gonzalez RE, Soto VC, Sance MM, Camargo AB, Galmarini $\mathrm{CR}$. Variability of solids, organosulfur compounds, pungency and health-enhancing traits in garlic (Allium sativum L.) cultivars belonging to different ecophysiological groups. J Agric Food Chem. 2009;57:10282-8. https://doi.org/10.1021/jf9018189

33. Sance MM, González RE, Soto VC, Galmarini CR. Relationships between antiplatelet activity, dry matter content and flavor in onion cultivars. J Food Agric Environ. 2008;6:41-6.

34. Morales-Soto A, García-Salas P, Rodríguez-Pérez C, JiménezSánchez C, Cádiz-Gurrea ML, Segura-Carretero A, Fernández-Gutiérrez A. Antioxidant capacity of 44 cultivars of fruits and vegetables grown in Andalusia (Spain). Food Res Int. 2014;58:35-46. https://doi.org/10.1016/j.foodres.2014.01.050

35. Balasundram N, Sundram K, Samman S. Phenolic compounds in plants and agri-industrial by-products: antioxidant activity, occurrence, and potential uses. Food Chem. 2006;99:191-203. https://doi.org/10.1016/j.foodchem.2005.07.042 